\title{
A biocompatible chitosan-ionic liquid hybrid catalyst for regioselective synthesis of $1,2,3$-triazols
}

\author{
Mansoureh Daraie, Majid M. Heravi \\ Department of Chemistry, School of Science, Alzahra University, PO Box 1993891176, Vanak, \\ Tehran, Iran
}

\begin{abstract}
A new type of quadruple hybrid system was prepared containing chitosan (CS), folic acid (FA), Poly(styrene-co-maleic anhydride) [SMA] and ionic liquid (IL). This nanocatalyst was synthesized by the functionalization of SMA with folic acid and then polymerization with chitosan in the presence of DCC and NHS. $\mathrm{CuI}$ was immobilized onto this support to provide $\mathrm{Cu} @$ SMA-FA-CS-IL. The catalyst was fully characterized by using different characterization techniques such as FTIR, 1H NMR, ICP, SEM, EDX, TGA, and XRD. This Cu (I) heterogeneous species was successfully applied in the reaction of sodium azide, terminal alkynes, and alkyl halides or $\alpha$-haloketones in water to afford 1,4disubstituted-1,2,3-triazoles within relatively short reaction times. Moreover, the catalyst is recyclable for five reaction cycles with minor $\mathrm{CuI}$ leaching and slight drop of the catalytic activity.
\end{abstract}

Keywords: Biopolymer, $\mathrm{Cu}$ (I) nanoparticles, Chitosan, SMA, Ionic liquid, Click reaction, 1,2,3-Triazoles 